\title{
ALGEBRAIC TOPOLOGICAL METHODS FOR CONTACT NETWORK ANALYSIS AND SYNTHESIS*
}

\author{
BY \\ CHARLES SALTZER \\ Case Institute of Technology \\ and \\ Electronics Laboratory, General Electric Company
}

1. Introduction. The use of Boolean algebra in the analysis and design of contact networks was introduced by C. Shannon [1], and for series-parallel circuits the problem was completely solved. However, for circuits of a more general nature, e.g., bridge networks, the design problem was not solved and the rules given for analysis depended on special geometric constructions carried out on the circuit diagram. The treatment of general circuits was approached from a topological point of view by the author [2] and S. Okada [3]. The method of Okada is analogous to the "loop" or "mesh" method of ordinary circuit analysis while the method of this paper is analogous to the "nodal" method [4] of ordinary circuit theory. It will be shown that contact network theory can be based on topological considerations and topological methods for analysis and synthesis will be given.

A contact network between two terminals is closed for a given state of the contacts if and only if there is a path between the two terminals such that each branch of the path is itself closed. With no loss of generality each branch may be regarded as containing a single contact and a branch is closed or open according to whether the contact is closed or open. The analysis problem consists of finding a formula for the state of the network in terms of the states of the contacts. The synthesis problem is: given such a formula, find a contact network which realizes it, subject usually to some sort of restriction such as minimality of the number of contacts.

The topological preliminaries will be given in Sec. 2. The analysis problem will be treated in Sec. 3 and the conversion of the topological formulas to formulas of Boolean algebra will be carried out in Sec. 4. The design problem will be treated in Sec. 5 and an example of the synthesis method is given in the last section.

2. Topological preliminaries. A graph is defined as a collection of segments or branches denoted by $x_{1}, x_{2}, \cdots, x_{b}$, and nodes which include the endpoints of the branches. The nodes will be denoted by $y_{1}, y_{2}, \cdots, y_{n}$. We consider linear forms in these variables choosing the coefficients from a modulo two arithmetic**, i.e.,

$$
\begin{aligned}
& 0+0=0 \\
& 0+1=1+0=1 \\
& 1+1=0 \\
& 0 \cdot 0=0 \\
& 0 \cdot 1=1 \cdot 0=0 \\
& 1 \cdot 1=1
\end{aligned}
$$

*Received May 15, 1958.

**The linear forms in the $x_{1} x_{2} \cdots x_{b}$ and the linear forms in $y_{1}, y_{2} \cdots y_{n}$ each form a group. 
The linear forms will be called 0-chains [5, 6], $C$, if the terms of the sum are nodes, and 1-chains, $\sigma$, if the terms are branches. The structure of a graph is known-and the graph can be drawn if the relation of the nodes to the branches is given. This information can be described by the boundary operator $\partial$ which is defined for segments by

$$
\partial x_{k}=y_{r}+y_{s},
$$

if $y_{r}$ and $y_{\mathrm{s}}$ are the endpoints of $x$, and for chains by linearity, i.e.,

$$
\partial\left(y_{k}+y_{r}\right)=\partial y_{k}+\partial y_{r} .
$$

These relations may also be expressed in matrix form by the incidence matrix (4) $A=\left(A_{r s}\right)$ where

$$
A_{r s}= \begin{cases}1 & \text { if } y_{s} \text { is an endpoint of } x_{r}, \\ 0 & \text { if not }\end{cases}
$$

The rows of this matrix correspond to branches and the columns to nodes. There are exactly two non-zero elements in each row in the matrix e.g., for Eq. (2), the kth row will have ones in the $r$ th and sth columns only. If the boundary of a chain is zero the chain will be said to be a cycle. In the bridge circuit of Fig. 1 the 1-chain $\sigma=x_{1}+x_{2}+x_{\star}$ is a cycle since $\partial \sigma=\partial x_{1}+\partial x_{2}+\partial x_{4}=y_{1}+y_{2}+y_{2}+y_{3}+y_{3}+y_{1}=0$.

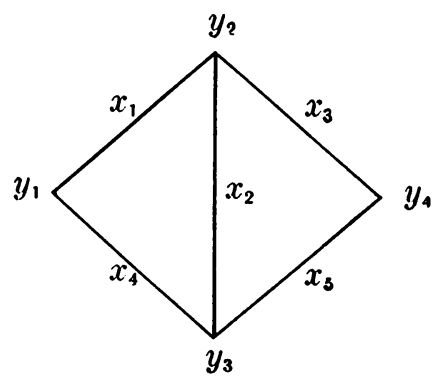

Frg. 1.

The boundary relations may be represented by

$$
\partial x_{r}=\sum_{s=1}^{n} A_{r s} y_{s} \quad(r=1,2, \cdots, b) .
$$

The incidence matrix for this bridge is:

$$
A=\left[\begin{array}{llll}
1 & 1 & 0 & 0 \\
0 & 1 & 1 & 0 \\
0 & 1 & 0 & 1 \\
1 & 0 & 1 & 0 \\
0 & 0 & 1 & 1
\end{array}\right]
$$

An alternative description is also given by the coboundary operator $\delta$ defined for nodes by

$$
\delta(\text { node })=\text { sum of the branches having this node as an endpoint, }
$$

and for chains by linearity, i.e., 


$$
\delta\left(y_{r}+y_{s}\right)=\delta y_{r}+\delta y_{s} .
$$

Thus, in Fig. 1

$$
\delta y_{2}=x_{1}+x_{2}+x_{3},
$$

and

$$
\delta\left(y_{1}+y_{2}\right)=x_{1}+x_{4}+x_{1}+x_{2}+x_{3}=x_{2}+x_{4}+x_{3} .
$$

The incidence matrix $A^{\prime}$, for this operator is defined by:

$$
A_{r \varepsilon}^{\prime}=\left\{\begin{array}{l}
1 \text { if } x_{\bullet} \text { has } y_{r} \text { as an endpoint } \\
0 \text { if not }
\end{array}\right.
$$

A comparison with (4) shows that $A^{\prime}$ is the transpose of $A$.

In terms of the matrix elements the coboundary operator may be represented by:

$$
\delta y_{r}=\sum_{s=1}^{b} A_{\imath r} x_{s} \quad(r=1,2, \cdots, n) .
$$

A chain is a cocycle if its coboundary is zero. For example, $C=y_{1}+y_{2}+y_{3}+y_{4}$ is a cocycle. A pair of nodes, $y_{r}$ and $y_{0}$ are said to be connected if there is a 1-chain $\sigma$ such that $\partial \sigma=y_{r}+y_{.}$. In Fig. $1, y_{1}$ and $y_{2}$ are connected since $\partial x_{1}=y_{1}+y_{2}$; and $y_{1}$ and $y_{4}$ are connected since $\partial\left(x_{1}+x_{3}\right)=y_{1}+y_{4}$. One such chain consists of the sum of the segments of any path connecting the nodes in question. Also, two nodes are said to be homologous if they are connected and when a graph is said to be connected it means that every pair of its nodes is connected. The lemma on which our considerations are based is:

Lemma. A zero chain is a cocycle if and only if, for every node in the zero chain, every node connected (homologous) to the given node is also present [6].

For completeness the proof is included. With no loss of generality we may consider only a connected graph. If the 0-chain consists of the sum of all the nodes then since every branch is a term of each of the coboundaries of exactly two nodes it is a consequence of the modulo-two addition that this chain is a cocycle. Conversely, if a node $y_{k}$ is in a cocycle then every node to which it is connected by a branch must also be in the cocycle; for if not, then the branch of which such a node is an endpoint would be present in the coboundary only once. In general, if $y_{r}$ is connected to $y_{k}$ then there is a chain which is a path $x_{a}+x_{b}+\cdots+x_{k}$ such that $\partial\left(x_{a}+x_{b}+\cdots+x_{k}\right)=y_{k}+y_{r}$, and every node which is an endpoint of a branch of this chain must be in the cocycle since, if this were not the case, some branch would appear only once in the coboundary. Hence, for any given node, the cocycle must contain every node connected to the given node.

3. Topological analysis of contact networks. An immediate consequence of the above lemma is

Theorem I. Two nodes of a graph are connected if and only if there is no cocycle which contains one of these nodes but not the other.

Thus, if we have a graph with $n$ nodes and say we wish to determine whether $y_{1}$ is connected with $y_{n}$, we form all the 0-chains which contain $y_{1}$ but not $y_{n}$-there are $2^{n-2}$ 
such chains-and find the coboundaries of these chains. If no coboundary vanishes, i.e., is a cocycle, then the two nodes are connected. We may indicate this symbolically by writing the formal product of these coboundaries with the convention that the product is to be zero if and only if at least one factor is zero, and our result may be stated as: $y_{1}$ and $y_{n}$ are connected if and only if the product of the given coboundaries is not zero.

An explicit formula can be written by listing the integers from zero to $\left(2^{n-2}-1\right)$ in binary form and prefixing zeros when necessary to make the total number of digits equal to $(n-2)$. We define the numbers

$$
\begin{aligned}
& a_{r s}=(s-1)^{\text {st }} \text { digit (counting from the left) of } r \text { in binary form } \\
& \quad\left[s=2,3, \cdots, n-1 ; r=0,1, \cdots,\left(2^{n-2}-1\right)\right] . \\
& a_{r 1}=1 \quad\left[r=0,1, \cdots,\left(2^{n-2}-1\right)\right] .
\end{aligned}
$$

All the 0-chains containing $y_{1}$ but not $y_{n}$ are

$$
C_{r}=\sum_{s=1}^{n-1} a_{r s} y_{s}, \quad\left(r=0,1, \cdots,\left(2^{n-2}-1\right) .\right.
$$

Hence the function $f$ which we shall call the state-function of the graph with respect to nodes $y_{1}$ and $y_{n}$ is

$$
f\left(x_{1}, x_{2}, \cdots, x_{b}\right)=\prod_{r=0}^{\left(2^{n-1}-1\right)} \delta C_{r},
$$

and is zero if and only if $y_{1}$ and $y_{n}$ are not connected.

By formula (8)

$$
\delta C_{r}=\sum_{s=1}^{n-1} a_{r s} \delta y_{s}=\sum_{s=1}^{n-1} a_{r s}\left(\sum_{k=1}^{b} A_{k s} x_{k}\right)
$$

Therefore,

$$
\delta C_{r}=\sum_{k=1}^{b}\left(\sum_{s=1}^{n-1} a_{r s} A_{k s}\right) x_{k}
$$

or

$$
\delta C_{r}=\sum_{k=1}^{b} \lambda_{r k} x_{k}
$$

where

$$
\lambda_{r k}=\sum_{s=1}^{n-1} a_{r s} A_{k s}, \quad\left[r=0,1, \cdots,\left(2^{n-2}-1\right) k=1,2, \cdots, b\right] .
$$

Thus in terms of the incidence matrix the state function $f$ can be written

$$
f\left(x_{1}, x_{2}, \cdots, x_{b}\right)=\prod_{r=0}^{\left(2^{n-1}-1\right)}\left(\sum_{k=1}^{b} \lambda_{r k} x_{k}\right) .
$$

For the circuit in Fig. 1 considered as a circuit between $y_{1}$ and $y_{4}$, the numbers in binary form from zero to $2^{n-2}-1=2^{2}-1=3$ are $00,01,10$, and 11 . Hence the numbers $a_{r}$, are given by 


$$
\left(a_{r s}\right)=\left[\begin{array}{lll}
1 & 0 & 0 \\
1 & 0 & 1 \\
1 & 1 & 0 \\
1 & 1 & 1
\end{array}\right]
$$

and the zero chains including $y_{1}$ but not $y_{4}$ are

$$
\begin{aligned}
& C_{0}=y_{1}, \\
& C_{1}=y_{1}+y_{3}, \\
& C_{2}=y_{1}+y_{2}, \\
& C_{3}=y_{1}+y_{2}+y_{3} .
\end{aligned}
$$

The coboundaries are

$$
\begin{aligned}
& \delta C_{0}=x_{1}+x_{4} \\
& \delta C_{1}=x_{1}+x_{2}+x_{5} \\
& \delta C_{2}=x_{2}+x_{3}+x_{4} \\
& \delta C_{3}=x_{3}+x_{5}
\end{aligned}
$$

and the state-function $f$ is

$$
f\left(x_{1}, x_{2}, \cdots, x_{5}\right)=\left(x_{1}+x_{4}\right)\left(x_{1}+x_{2}+x_{5}\right)\left(x_{2}+x_{3}+x_{4}\right)\left(x_{3}+x_{5}\right) .
$$

Precisely the same state-function except for the order of the factors is obtained by excluding $y_{1}$ and including $y_{n}$ in all the zero chains. This can be seen from the fact that the desired 0-chains can be obtained by adding $y_{1}+y_{n}$ to the given chains, since by the modulo-two addition this replaces $y_{1}$ by $y_{n}$. The coboundaries of these chains are, therefore, obtained from the coboundaries of the given chains by adding $\delta\left(y_{1}+y_{n}\right)$. Since the sum of all the nodes is a cocycle we have $\delta\left(y_{1}+y_{n}\right)=\delta\left(\sum_{k-2}^{n-1} y_{k}\right)$. Hence we can get the desired coboundaries from the given ones by adding $\sum_{k-2}^{n-1} y_{k}$ to the given 0 -chains instead of $y_{1}+y_{n}$. The numbers $a_{r s}$ which we get by this computation are obtained from the binary representation of $r$ by adding one to each of the $(n-2)$ rightmost digits of $r$ modulo-two. The resulting sums must be distinct since if they were not, the original numbers would not have been distinct. Since there are $2^{n-2}-1$ of these, this list must be a permutation of the original list and hence the new $\left(\lambda_{r s}\right)$ matrix is a row-permutation of the original and we get the same state-function.

We define a subgraph of a given graph as the graph obtained by deleting any branches (but not the nodes of these branches) from the given graph; and we have

Theorem II. The state-function $f$ of a subgraph with respect to a given pair of nodes is obtained from the state-function $f$ of the given graph with respect to the same nodes by setting all the branches not in the subgraph equal to zero (or deleting them) in the state-function of the given graph.

The incidence matrix of the subgraph is obtained from the incidence matrix of the graph for the boundary operator by replacing the ones in the rows corresponding to the deleted branches by zeros, and the coboundaries of the nodes with respect to the sub- 
graph are obtained from the coboundaries of the nodes with respect to the graph by setting them equal to zero (or deleting them) in the latter. Hence the coboundaries of the 0 -chains $C_{r}$ with respect to the subgraph are obtained by setting the deleted branches of the graph equal to zero in the coboundaries of the 0-chains of the original graph. Consequently by the definition of the state-function, the theorem is proved.

In the graph of Fig. 1, for example, if we delete branches $x_{1}$ and $x_{4}$ the incidence matrix $\alpha_{s}$ of the subgraph becomes

$$
\bar{A}_{r s}=\left[\begin{array}{llll}
0 & 0 & 0 & 0 \\
0 & 1 & 1 & 0 \\
0 & 1 & 0 & 1 \\
0 & 0 & 0 & 0 \\
0 & 0 & 0 & 1
\end{array}\right]
$$

the coboundaries become

$$
\begin{aligned}
& \delta C_{0}=0, \\
& \delta C_{1}=x_{2}+x_{5}, \\
& \delta C_{2}=x_{2}+x_{3}, \\
& \delta C_{3}=x_{3}+x_{5}
\end{aligned}
$$

and the state-function becomes

$$
f_{s}\left(x_{2}, x_{3}, x_{5}\right)=f\left(0, x_{2}, x_{3}, 0, x_{5}\right)=0,
$$

and we see that nodes $y_{1}$ and $y_{4}$ are not connected.

This interpretation of the formula can be stated as

Theorem III. The connection between two nodes is zero when a given set of branches is opened (deleted) if and only if this set contains all the branches in at least one of the 0-chain coboundaries. Conversely, the given nodes are connected if and only if at least one branch in each of the 0-chain coboundaries is not opened provided the state-function is not identically zero.

This theorem shows how to write a state-function from the conditions of operation.

We define equivalent state-functions as functions which are zero for precisely the same sets of branch states and Theorem III has as a consequence a minimization method, namely,

Theorem IV. If the coboundary of any 0 -chain, i.e., factor of $f$, is contained in another factor then the state function obtained from the given state-function by deleting the first factor is equivalent to the given state-function.

It is clear that the results of combinatorial switching theory can be recovered from this point of view.

As the last two examples we take branches in series and parallel.

For the series network of Fig. 2(a) the above method yields $f\left(x_{1}, x_{2}\right)=x_{1} x_{2}$ and for the parallel network of Fig. 2(b), $f\left(x_{1}, x_{2}\right)=x_{1}+x_{2}$. As in the method of Boolean 


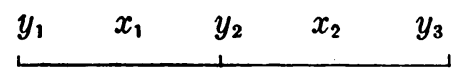

(a)

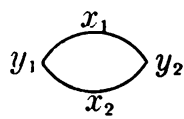

(b)

Fig. 2.

algebra, if we consider the states of the branches as being determined by individual state-functions, we can write the state functions of series-parallel networks directly by inspection.

Thus, in addition to the explicit formulas for the states of general networks the preceding theorems show that the theory of contact networks can be founded on an algebraic topological basis. It is not suggested that this approach be a substitute for the methods of Shannon but rather a supplement, and in the next section we consider the conversion of these formulas into the formulas of Boolean algebra.

4. The reduction of state formulas of Boolean algebra. Following Shannon (1.c.) we introduce the Boolean elements $0^{*}$ and $1^{*}$ where

$$
\begin{aligned}
& 0^{*} \oplus 0^{*}=0^{*}, \\
& 0^{*} \oplus 1^{*}=1^{*} \oplus 0^{*}=1^{*} \oplus 1^{*}=1^{*}, \\
& 0^{*} \cdot 0^{*}=0^{*} \cdot 1^{*}=1^{*} \cdot 0^{*}=0^{*}, \\
& 1^{*} \cdot 1^{*}=1^{*} .
\end{aligned}
$$

The admittance between two nodes is $1^{*}$ if the circuit is closed and $0^{*}$ if the circuit is open. Also if $\beta$ is a Boolean variable which assumes the value $1^{*}$ when a given relay is operated and $0^{*}$ otherwise, then the states of the normally open contacts of the relay are denoted by $\beta$ and the states of the normally closed contacts are denoted by the complement of $\beta$, i.e., $\bar{\beta}$. If we denote the state of the branch $x_{k}$ of the graph of the contact network by $\alpha_{k}$ then the state-function is transformed into a Boolean function and we note that the value of this Boolean function is $0^{*}$, if and only if at least one factor is $0^{*}$. A factor is $0^{*}$ if and only if each of its terms is $0^{*}$. By comparison with the results of the preceding section we see that the value of this Boolean function is $0^{*}$ if and only if the circuit between the given nodes is open. Consequently, if the circuit is closed each factor has the value $1^{*}$, and the value of the Boolean function is $1^{*}$. This completes the proof of

Theorem V. The Boolean admittance function for a pair of terminals of a network is obtained from the state-function with respect to those terminals by replacing the branches by their admittances.

The calculations are simplified if the columns of the incidence matrix $A$ are used as column vectors representing the coboundaries.

It may also be noted that the admittances between different pairs of nodes of a network are related in a simple way so that if we have one state-function we can get the others immediately. This can be seen from the following considerations. Assume that the factors of the state-function with respect to terminals $y_{1}$ and $y_{n}$ are known. Assume that these have been obtained by including $y_{1}$ in all the 0 -chains since we get the same results by using either $y_{1}$ or $y_{n}$. To compute the state-function between $y_{1}$ and $y_{k}$ we 
need the 0-chains which include $y_{1}$ but not $y_{k}$, i.e., we must replace $y_{k}$ in the first set of 0 -chains by $y_{n}$. Since $y_{k}$ occurs in $C_{r}$ multiplied by $a_{r k}$, the replacement can be effected by adding $a_{r k}\left(y_{k}+y_{n}\right)$ to $C_{r}$. Hence the factors of the desired state-function are obtained by adding $a_{r k}\left(\delta y_{k}+\delta y_{n}\right)$ to $\delta C_{r}$. A similar argument holds for replacing node $y_{1}$.

Theorem VI. The state-function for the nodes $y_{i}$ and $y_{i}$ can be obtained from the statefunction for the nodes $y_{i}$ and $y_{k}$ by adding $a_{r k}\left(\delta y_{k}+\delta y_{i}\right)$ to the factors $\delta C_{r}$ of the given state-function.

The matrix of these state-functions when the branches are replaced by their admittances is the admittance matrix of Lunts [7] if the diagonal elements are taken as $1^{*}$.

5. The synthesis problem for a two-terminal network. A method for finding general networks, i.e., not necessarily series-parallel networks, which realizes the graph of a contact network for a given two-terminal Boolean admittance will be given in this section. We proceed by reversing the analysis in Secs. 3 and 4 . The first step is to write the given function as a product of sums of admittances. The second step is to assign branches to each admittance. At this stage there may be a considerable number of possibilities. One extreme is to assign a different branch to each appearance of a contact admittance or its complement; the other extreme is to assign the same branch to each appearance of a variable. The third step consists of writing a state-function by replacing the admittance variables by the corresponding branch variables. The fourth step is to select an integer $n$ such that $b+1$ is greater than $n$ and $2^{n-2}$ is not less than the number of factors of the state-function.

In the fifth step if $2^{n-2}$ is greater than the number of factors of the state-function, then factors subject only to the restriction that the terms of such a factor contain at least the set of nodes in one of the given factors are added so the total number of factors is $2^{n-2}$. The sixth step is to assign a number $r\left[r=0,1,2, \cdots\left(2^{n-2}-1\right)\right]$ to each of the factors. In the seventh step the equations for the elements of the incidence matrix are written using Eq. (12). The last step is to solve these equations. If the solution has the property that there are no more than two non-zero elements in any row of the solution displayed in matrix form, i.e., is a partial incidence matrix, a column is added so that an element of this column is 0 if there are two ones or no ones in this row, and 1 if there is only one 1 in this row. If the partial incidence matrix does not satisfy the above condition then the computations can be repeated for possibly different assignments of $r$, $b, n$, and correspondences of the admittances and branches. An example will be given below. That the above problem has at least one solution is obvious since a series-parallel network can always be drawn.

To clarify the method we retrieve the bridge network of Sec. 1 where $f\left(\alpha_{1}\right.$, $\left.\alpha_{2}, \cdots, \alpha_{5}\right)=\left(\alpha_{1}+\alpha_{4}\right)\left(\alpha_{1}+\alpha_{2}+\alpha_{5}\right)\left(\alpha_{2}+\alpha_{3}+\alpha_{4}\right)\left(\alpha_{3}+\alpha_{5}\right)$. We choose $n=4$, $b=5$ and replace $\alpha_{k}$ by $x_{k}$. The numbers $r=0,1,2,3$ are assigned so that

$$
\begin{aligned}
& \delta C_{0}=x_{1}+x_{4} \\
& \delta C_{1}=x_{1}+x_{2}+x_{5} \\
& \delta C_{2}=x_{2}+x_{3}+x_{4} \\
& \delta C_{3}=x_{3}+x_{5} .
\end{aligned}
$$


Thus the matrices $A$ and $\lambda$ are given by

$$
a=\left(a_{r s}\right)=\left[\begin{array}{ccc}
1 & 0 & 0 \\
1 & 0 & 1 \\
1 & 1 & 0 \\
1 & 1 & 1
\end{array}\right], \quad \lambda=\left(\lambda_{r k}\right)=\left[\begin{array}{ccccc}
1 & 0 & 0 & 1 & 0 \\
1 & 1 & 0 & 0 & 1 \\
0 & 1 & 1 & 1 & 0 \\
0 & 0 & 1 & 0 & 1
\end{array}\right]
$$

and the system of equations (12) becomes

$$
\begin{array}{r}
A_{k 1}=\lambda_{0 k}, \\
A_{k 1}+A_{k 3}=\lambda_{1 k}, \\
A_{k 1}+A_{k 2}=\lambda_{2 k}, \\
A_{k 1}+A_{k 2}+A_{k 3}=\lambda_{3 k} . \\
(k=1,2,3,4,5)
\end{array}
$$

The solution of this system is

$$
\begin{aligned}
& A_{k 1}=\lambda_{0 k}, \\
& A_{k 2}=\lambda_{0 k}+\lambda_{2 k}, \\
& A_{k 3}=\lambda_{0 k}+\lambda_{1 k},
\end{aligned}
$$

which in matrix form is the partial incidence matrix

$$
\left[\begin{array}{lll}
1 & 1 & 0 \\
0 & 1 & 1 \\
0 & 1 & 0 \\
1 & 0 & 1 \\
0 & 0 & 1
\end{array}\right] .
$$

We get the incidence matrix by adding a column which brings the number of ones in each row to two,

$$
\left[\begin{array}{llll}
1 & 1 & 0 & 0 \\
0 & 1 & 1 & 0 \\
0 & 1 & 0 & 1 \\
1 & 0 & 1 & 0 \\
0 & 0 & 1 & 1
\end{array}\right]
$$

which is the original matrix. The contact network can be drawn immediately.

The equations for the general case may be restated as 


$$
\sum_{s=1}^{n-1} a_{r s} \delta y_{s}=\delta C_{r} \quad\left[r=0,1, \cdots,\left(2^{n-2}-1\right)\right] .
$$

From the definition of $a_{r s}$ we see that if the system has a solution it must be

$$
\begin{gathered}
\delta y_{1}=\delta C_{0} \\
\delta y_{2}=\delta C_{0}+\delta C_{2^{n-s}}, \\
\cdots \cdots \cdots \cdots \cdots \cdots \cdots \cdots \cdots \cdots \\
\delta y_{k}=\delta C_{0}+\delta C_{2^{n-k-1}} \\
\cdots \cdots \cdots \cdots \cdots \cdots \cdots \\
\delta y_{n-1}=\delta C_{0}+\delta C_{1}
\end{gathered}
$$

We call these chains a reduced set of factors. Since the ordering of the factors in the state-function is at our disposal we may determine solutions by choosing one of the factors as $\delta C_{0}$ and adding this factor to each of the others. If from these $\left(2^{n-2}-1\right)$ chains we can select $(n-2)$ chains such that no branch occurs more than twice as a term in the set of chains consisting of these $(n-2)$ chains together with the chain designated as $\delta C_{0}$, then we have a partial incidence matrix. The consistency of the set of equations (14) must of course be verified by substitution of the trial solution.

Thus for a given assignment of branches, nodes, and order of factors the synthesis problem for a given state-function can be solved if and only if the system of equations (14) is consistent and the set of coboundaries (15) satisfies the condition that no branch appears more than twice.

The consistency equations (14) may be regarded from another point of view. If we add $\delta C_{0}(\bmod 2)$ to each of the equations of (14), the resulting system is generated from the $(n-2)$ sets $\delta y_{2}, \delta y_{3}, \cdots \delta y_{n-1}$ by taking all possible sums, i.e., the set $\left(\delta C_{0}+\delta C_{k}\right)$ $\left[k=0,1,2, \cdots\left(2^{n-2}-1\right)\right]$ is isomorphic to a Boolean algebra with $(n-2)$ atoms. The determination of whether or not this is the case can be shown to be independent of the choice of $\delta C_{0}$, and since the order of the factors of the state-function can be assigned arbitrarily, the consistency can also be shown to be independent of the ordering of the factors. Thus the test of consistency requires only that an arbitrarily selected factor be added to all the factors and any $(n-1)$ of these sums (modulo 2 ) be chosen as atoms. If each of these sums is a linear combination of the atoms then the set of equations (14) is consistent, otherwise the system is inconsistent and the assignment of branches to the admittances must be made in another way.

If the number of factors of the state-function is less than $2^{n-2}$ then the additional factors have the admittance one when the circuit is closed. Thus these factors must arise from branches whose admittances are complementary or must contain as a subset the branches which are present in another factor. This can be verified by generating the complete reduced set $\left(\delta C_{r}+\delta C_{0}\right)\left(r=1,2, \cdots, 2^{n-2}-1\right)$ adding $\delta C_{0}$ and verifying the above condition. It follows that for a given assignment of branches and choice of $n$ the verification of the consistency equations (14) can be made independently of the choice of $\delta C_{0}$, i.e., holds for all choices, and when the consistency equations are satisfied the problem is reduced to determining whether an assignment of the factors of the state-function can be made so that each branch occurs no more than twice in these factors. This may not be the case even for a consistent set.

It may be remarked that the complexity of this method is due in part to the fact that all circuits realizing the given function can be obtained this way. 


\section{REFERENCES}

1. C. E. Shannon, A symbolic analysis of relay and switching circuits, Trans. A.I.E.E. 57, 713 (1938)

2. C. Saltzer, Two-terminal relay and switching circuits, (Abstract) Bull. Am. Math. Soc. p. 347 (1954)

3. S. Okada, Topology applied to switching circuits, Proc. of the Symposium on Information Networks, New York, p. 267, (1954)

4. C. Saltzer, The second fundamental theorem of electrical networks, Quart. Appl. Math. XI, 119-123 (1953)

5. O. Veblen, Analysis situs, Am. Math. Soc. Colloquium Publications 5, part 2, New York, 1922

6. B. Eckman, New York University Notes on Topology (mimeographed notes)

7. A. G. Lunts, The application of Boolean matrix algebra to the analysis and synthesis of relay contact networks, Doklady Akad. Nauk, SSR, 70, 421-423 (1950) 